\title{
Agent-Based Model Heuristics in Studying Memory Mechanisms
}

\author{
Alexandr A. Ezhov1, Svetlana S. Terentyeva ${ }^{2}$ \\ ${ }^{1}$ Troitsk Institute for Innovation and Fusion Research, Moscow, Russia \\ ${ }^{2}$ Alfa-Bank, Moscow, Russia \\ Email: ezhov@triniti.ru
}

Received 1 February 2014; revised 1 March 2014; accepted 28 March 2014

Copyright (C) 2014 by authors and Scientific Research Publishing Inc.

This work is licensed under the Creative Commons Attribution International License (CC BY). http://creativecommons.org/licenses/by/4.0/

(c) (i) Open Access

\section{Abstract}

This study is aimed at proposing an agent-based model formulated in (Ezhov \& Khrennikov, 2005) and describing the emergence of specific binary memory codes in the system characterized by high inequality. The model is offered as a heuristic tool to predict a new competitive pair in the human brain's memory localized in the hippocampus and prefrontal cortex, correspondingly. This model is able to account for the influence of sociological factors to the brain memory.

\section{Keywords}

Agent-Based Model, Inequality, Memory Codes, Hippocampus, Prefrontal Cortex

\section{Introduction}

It is widely known that any finding in memory mechanisms used in brain can be naturally applied by engineers and scientists to development of new powerful intelligent technical systems. An opposite situation can be considered-Is it possible to use the results of mathematical modeling of complex systems to guess some properties of biological systems? Apart from such connectionist models as neural networks there exist some agent-based systems having in-built memory or memory arising as an emergent property of a complex system. The crucial fact is that such systems can take into account some sociological or economic factors that can influence some memory phenomena (Krupa, 2009). In this paper, we analyze the possibility to use the agent-based model proposed in (Ezhov \& Khrennikov, 2005) to make some predictions about possible memory mechanisms implemented by human brain. One of these predictions is that along with the competition between such brain structures as the hippocampus and the striatum performing declarative and non-declarative memories there can also be a competition between memories represented in the prefrontal cortex and the hippocampus in different brain hemispheres. It is suggested that in the situation of high unfairness or inequality this competition should lead to 
the formation of winners in the right brain hemisphere- the right dorsolateral prefrontal cortex (this is supported by the experiments of D. Knoch et al., 2006) and the left hippocampus in the left brain hemisphere. If these conclusions or their possible analogies (which can differ in some other localization of the memory systems considered) are justified the model proposed in (Ezhov \& Khrennikov, 2005) may be useful for some brain memory system simulations. Otherwise, it can be evidently rejected as having no or little relation to memory modeling. In any case, we understand that any theoretical model has a real value only if it is able not only to describe known facts, but also to predict new phenomena.

The structure of the article is as follows. In the first part, we outline briefly the importance of the application of human-like agent models to the analysis of collective phenomena emerging in a complex society and especially in an unequal society. In the second part, the agent-based model proposed in (Ezhov \& Khrennikov, 2005) is described and the emergence of the specific memory codes is discussed. In the third part, the connection of these results with modeling an alcohol abstinent syndrome is discussed. In the last part, the model is applied to make a suggestion about the brain structures which use these specific memory codes in the conditions of big inequality in the proposal of material resource in different social niches (e.g. professional), namely, left hippocampus and right dorsolateral prefrontal complex. This can open the way to elaborate theoretical models which are able to take into account sociological factors when studying brain memory mechanisms.

\section{Agent-Based Models of Complex Systems}

The agent-based approach is widely used to study complex systems (Guckenheimer et al., 2008: pp. 2, 16). This is because a set of well-elaborated mathematical and physical approaches have failed when applied to systems with uncertain future. The agent-based model to be considered here was introduced and studied in (Ezhov \& Khrennikov, 2005). In this toy model an artificial world consists of cells which, in general, can be populated by an arbitrary number of agents. Complex systems consist of many units which interact with each other in a nonlinear manner. It means that the effect of their action is not proportional to its value. Main features of complex systems are emergent patterns and emergent properties. The emergent patterns are surprisingly stationary, oscillating or moving objects which cannot be predicted from the first principles. The emergent properties (e.g., an ability to perform universal computations) describe the system as a whole which (in the case when the system is really complex) is more than simply a sum of its parts.

Patterns of economic and social inequality can be considered as emerged global characteristics of a complex society. The human identity itself can be also regarded as a complex system involving nationality, culture, language, ethnicity, ethic, religion, profession, etc. We can treat identity as a set of these self-descriptive and interconnected characteristics of a human being. For using agent-based models in sociology, economy, etc. of necessity is to have a realistic single-agent model of human. Surely, the human brain is also a complex system, but the point is that the brain is asymmetrical. Instead of this word physicists prefer to use the term broken symmetry. The symmetry breaks when some of the system parameters, e.g., a temperature, changes (as is observed at water-to-vapor transition). Phase transitions can be connected (or not) with the fact of symmetry breaking.

In brain studies it is important to guess how economic and social inequality can influence brain and memory. It has been shown earlier that, as it is in physics, the phase transitions caused by growth of inequality can be observed (Ezhov, Khrennnikov, \& Terentyeva, 2008: pp. 031126-7). The result of this work shows that it is possible to advance a hypothesis about a human memory asymmetry in highly unequal societies. It means that different types of memory and memory codes can be attributed to different brain hemispheres. It also implies that, in some sense, no individual memory exists and a complex society consisting of individuals is, in fact, a nested system, i.e. it consists of complex agents. The same can be said about a collective memory (it is nested), i.e., it is produced by factually multiple memories of individuals (Squire, 2004).

The point is that according to the model (Ezhov \& Khrennikov, 2005), the key parameter responsible for the emergence of memory asymmetry, its binary structure and cognitive technology of counting is inequality. It is well-known, that social and economic inequality characterizes the difference both between people and countries. In recent years many papers devoted to the question of people reaction to inequality have been published.

It is remarkable that the economic mainstream-neoclassical economics-has failed to explain the form of the wealth distribution curve. This has been was successfully described by physicists only recently (Scafetta, Picozzi, \& West, 2004: p. 353). Besides, the human behavior in situations of an unjust distribution of wealth is contrary to the main feature of a rational agent of neoclassical economics-homo oeconomicus - the utility 
maximization. This was confirmed by the results of studying the classical Ultimatum Game (Thaler, 1988). When playing in reality, the players reject unjust splitting of a potential prize by giving preference to the case of receiving nothing but not an unjust part. It is also notable that this rejection of any unequal sharing can be suppressed by applying a magnetic field to the right prefrontal cortex of player brain (Knoch et al., 2006: p. 6469).

In 2010, the study of frontal parts of striatum revealed a strong aversion to a highly unequal payment for equivalent job: the aversion was expressed by human who received both excessive and insufficient payments (Tricomi, 2010: p. 1089). By studying the key factors of the economic growth, it was found that any improvement of law systems, property rights, or democracy is of no use if a sharp inequality in society exists (Easaw, 2010: p. 142).

Therefore, when building realistic economic and social models, it is extremely important that the inequality level should be taken into account. Such models are being developed in the growing field of econophysics (Mantegna \& Stanley, 2000). Physics is the basic discipline for this field which treats society as a complex system and does not use the optimum principle to describe behavior of agents. In some sense human beings in such models may be considered analogously to atoms which are able to attract or repulse each other.

\section{Agent-Based Model of Unequal Society}

In the model proposed in (Ezhov \& Khrennikov, 2005) the artificial world consists of cells which, in general, can be populated by an arbitrary number of agents. The crucial feature of an artificial agent in the model is it has two kinds of resources: physical and mental ones. The physical resource is necessary for the agent to be alive physically, so the resource can be treated as food, water, etc. The second one corresponds to the agent's identity -the complex of its self-identification as belonging to a given nation, religion, profession, country, etc. The agent dies if any of its resources expires. Its death can be either physical, or mental. The latter corresponds to full loss of the agent's identity. It is supposed that the world is divided into cells and the initial cell where the agent starts its life corresponds to its given identity.

If an agent changes the cell it occupies, its identity also changes though not fatally, in general, but, if the number of changes exceeds a certain threshold, the agent can die mentally. On the other hand, the agent is provoked to change its identity by changing the cell because the food needed to survive physically can be presented in another cell. So, to survive mentally the agent should hold its cell, if possible, but to survive physically it should change its cell, because the necessary food can appear in other cells. The point is that the probability to receive a proposal for food depends on the cell and the distribution of this probability across the cell is highly non-uniform, in general. Preventing both resources from vanishing (mental and physical surviving) turns out to be a contradictory problem. The crucial model feature which connects this with the field of statistical physics is that inequality in the food proposal in a different cell can be described by an effective temperature of environment. So a high temperature corresponds to a low level in inequality of food presentation in different cells, while a low temperature- to a highly unequal presentation of it across these cells (Figure 1).

Then, it is expected that if an agent changes its cell to consume food, its mental resource decreases. We interpret this situation as if the agent consumed its mentality to solve the problem of physical survival. We also suggest that the agent could not increase or compensate its mental resource by any means. Surely, if food appears in the agent's cell, it can be consumed without changing the agent's mental resource. It is necessary to further clarify our concepts of the world cells, or identity and mental surviving. One should not consider the world cells as those of some physical space. They do not have neighbors or far located cells, so no distance between them is introduced. Each of the world cells can be characterized by a set of parameters used by human for self-identification.

We interpret the appearance of food in the cell being free of a specific agent as the environment proposal either to change the identity of the latter or, that is the same, to pay a unit of mental resource for the food. For non-interacting agents there are only two reasonable strategies to survive. Following the first one, the agent keeps its mental resource regardless of the cell in which the food is proposed. As this food can occasionally appear in the cell of the agent, the latter has also a chance to survive physically if such a lucky event happens frequently enough. Following the second reasonable strategy, the agent always accepts environmental proposals for increasing its physical resource by food consumption. The agent also has a chance to keep its mental resource when the environment does not demand to change the cell of its location.

These two strategies were called in (Ezhov \& Khrennikov, 2005) the right brain and left brain strategies, cor- 

Low inequality
High temperature $\mathrm{T}_{1}$
High inequality
Low temperature $\mathrm{T}_{2}<\mathrm{T}_{1}$
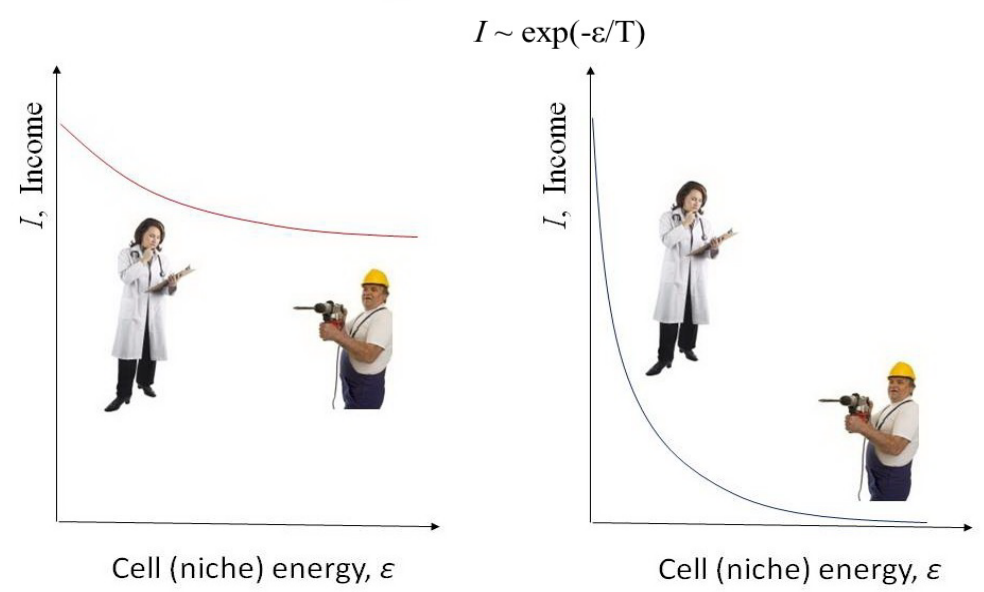

\begin{abstract}
Figure 1. Two cases of an unequal cellular world with different distribution of food (income) proposal in different cells (niches, or professional identities). Each cell is characterized by effective energy which grows from a well-paid to the lowest one. Left: Low level of inequality is characterized by high effective temperature. In this case the incomes of the doctor and worker do not differ substantially. Right: High level of inequality corresponds to a low effective temperature. In this case the income of a doctor is remarkably higher than that of a worker.
\end{abstract}

respondingly. There is a real basis to relate such kinds of behavior with the dominance of the corresponding brain hemisphere. It follows from the concrete exponential form of logical functions representing these strategies which were used by Lefebvre in his algebra of consciousness (Lefebvre, 2001: p. 47), actually from the absence and presence of logical negation in them. Indeed, there exists some experimental evidence that the right hemisphere is unable to create logical negations: all logical operations are functions of the left hemisphere.

Some other arguments in favor of this interpretation of the two reasonable strategies are available. One of the arguments was presented in (Rotenberg \& Arshavsky, 1997: p. 49). The authors suggested that “... in its most general form the difference between the two strategies of thinking is reduced to opposite modes of organizing the contextual connections between elements of information. 'Left-hemisphere' mode of thinking so organizes any sign material (whether symbolic or iconic) as to create a strictly ordered and unambiguously understood context. Its formation requires an active choice, out of the real and potential connections between the multiform objects and phenomena of a few definite connections, which would not create internal contradictions (!) and would facilitate an ordered analysis... In contrast, the function of 'right-hemispheric', 'image' thinking is a simultaneous capture of an infinite number of connections and the formation due to this capture of an integral but ambiguous context. In such a context, the whole is not determined by its components since all specific features of the whole are determined only by connections between these parts. On the contrary, any concrete element of such a context bears a determining stamp of the whole. A new experience is incorporated in this holistic picture of the world. Individual facets of images interact with each other on many semantic planes simultaneously. Examples of such contextual connections are the connections between images in sleep dreams or in work of art. The advantages of this strategy of thinking manifest themselves only when the information itself is complex, internally contradictory and basically irreducible to an unambiguous context.” (Rotenberg \& Arshavsky, 1997: p. 50).

The most interesting behavior of agents can be in the situation where they can interact mentally when making decision to hold or change their identity. Concrete forms of this interaction were obtained by making a general proposal that the left brain dominance corresponds to the first (Western) ethical system, while the right brain dominance- to the second (Soviet) ethical system. These two fundamentally different ethics were discovered and described by Lefebvre. He has demonstrated (Lefebvre, 2001: p. 42) that it is possible to construct two algebraic models of ethic which differ from each other in different interpretation of two basic operations_adding 
and multiplication. They can be interpreted as confrontation and cooperation, correspondingly, or vice versa (Lefebvre, 2001: p. 44). It is remarkable, that these two variants of ethic based on these interpretations adequately represent the results of the experiments performed in USA with some emigrants from Europe (1st ethic system) and the Soviet Union (2nd ethic system).

In short, the first ethic system is based on formal prohibition of evil, while the second one on informal declaration of good. This is sufficient to connect formal thinking inherent to the left brain hemisphere with the first ethic system and informal contradictory and dialectical thinking with the second ethic system. This link permits using the Lefebvre formalism to infer general interaction rules for both left and right brain dominant agents.

By doing so, it was possible to show that the cooperative right brain dominant agents effectively attract each other, while the competitive left brain dominant ones effectively repulse each other (in the case of self-interaction an agent changes its dominant hemisphere). As follows from (Ezhov \& Khrennikov, 2005: p. 016138-7) the equilibrium state of the collective of cooperative right brain agents is described by a well-known statistics of Bose-Einstein. In physics this statistics describes particles called the bosons (including photons and, e.g. atoms of Helium-4). At a macroscopic level the ensembles of bosons can demonstrate many remarkable quantum properties such as superfluidity or laser coherent irradiation. The equilibrium state of the left brain dominant agents is described by some variant of the Fermi-Dirac statistics-nuclear core particles such as protons and neutrons, as well as electrons in atom are described by this kind of statistics (Figure 2).

More information about the model properties can be revealed not from equilibrium distributions, but from the study of fluctuations, i.e. temporal deviations of agent-in-cell numbers from their equilibrium mean values. This

$$
\begin{aligned}
& \text { Consumption preference } \\
& \qquad \psi(a)=a \neg a \\
& \text { Antisymmetrical function }
\end{aligned}
$$

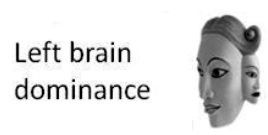

$1^{\text {st }}$ ethical system

repulsion (competition)

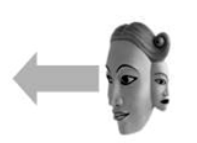

Fermi-Dirac-like

cell occupation

$$
n_{i}=\frac{N-1}{e^{\left(\varepsilon_{i}-\mu\right) / T}+1}
$$

1

2

4

$$
\begin{aligned}
& 2^{\text {nd }} \text { ethical system } \\
& \text { attraction (cooperation) }
\end{aligned}
$$

5

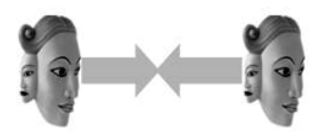

Bose-Einstein-like cell occupation

6

Figure 2. Two basic agent strategies differ in the priority of material food consumption and mental resource (identity) preservation correspondingly 1), in the description using the asymmetrical and symmetrical Boolean functions which define the agent decision $\psi=0$ to accept the environment proposal to change the cell ( $a=0$ ) or to hold it ( $a=1, \psi=1) 2$ ). These two functions are connected with the operation of the left (logical) and right (intuitive) brain hemispheres. The operation of these hemispheres was related with the formalism of Lefebvre's first and second ethical systems 4). The use of the last formalism to describe the agent interaction leads to a repulsive character of the competitive left brain dominant agents and to an attractive character of the cooperative right brain dominant agents 5). As a result, the equilibrium distribution of left brain agents turns to be fermion-like, while the equilibrium distribution of the right brain agents turns to be boson-like. 
demands to look attentively at the dynamics of agent movement through their identity world cells. It is very important that we can face only three different events in this dynamics. Indeed it is possible to follow the histories of agents in the systems under condition of thermodynamic equilibrium. Let us call the choice of the destination cell where the material resource (food) is offered to the agent as "the proposal" to go to this cell. The agent can either accept or reject this proposal. According to its decision the agent can either stay in the initial cell or go to the destination cell. Now we can define three different situations which can occur:

- The destination cell differs from the current cell of the agent and the agent accepts the proposal to go to a new cell. We can denote this situation using a pair of characters AG (accept and go).

- The destination cell differs from the current cell of the agent, but the agent rejects this proposal and stays in the previous cell. We can denote this situation using a pair of characters RS (reject and stay).

- The destination cell coincides with the current agent cell. In this case the agent unconditionally accepts the proposal and stays in the previous cell. We can denote this situation using a pair of characters AS (accept and stay).

By collecting the events happened to the given agent we can form its history which can be represented by a three-symbol ternary code $\left(\mathrm{h}_{1}, \mathrm{~h}_{2}, \mathrm{~h}_{3}\right)$ assuming that $\mathrm{h}_{1}=\mathrm{AG}, \mathrm{h}_{2}=\mathrm{RS}, \mathrm{h}_{3}=$ AS. The next step is connected with the derivation of two memories from a single history. The memories are obtained by partial forgetting this history. Indeed, we can easily derive two special binary memory codes by omitting the second or the first character in the ternary history code. The first choice gives characters $A$ and $R$-we call this code $\{A, R\}$. The second choice leaves characters $S$ and $G$ and we call this code $\{S, G\}$ (Ezhov \& Khrennikov, 2006: p. 60). If we describe the memory of the given agent using the $\{\mathrm{A}, \mathrm{R}\}$ or $\{\mathrm{S}, \mathrm{G}\}$ codes, we may evidently lose some information about the true history. In particular, if any agent accepts the proposal (A), it can either stay in the initial cell or go to another one. On the other hand, if the agent stays in the initial cell, this implies that it either accepts the proposal to stay there or rejects the proposal to go to another cell. Therefore, it is evident that such two binary coding schemes for memory are not equivalent: it is impossible to reconstruct the $\{\mathrm{A}, \mathrm{R}\}$ agent memory given the $\{\mathrm{S}, \mathrm{G}\}$ memory and vice versa. Both of these coding schemes can be considered as a projection of the full history coding on two orthogonal planes.

Why can these two binary codes define memory code variants? Because there is some evidence that the brain can really work with the binary codes only (Lin, Osan, \& Tsien, 2006: p. 49). As it was shown in (Ezhov \& Khrennikov, 2006: p. 59), the possibility of coding the agent memories by use of two different schemes opens the way to relate collective memories of the right and left brain dominant agents by calculating the degree of ultrametricity (Murtagh, 2006: p. 263) for sets of their incomplete binary coded memories (Figure 3). Briefly speaking to calculate the degree of ultrametricity of a set of agent histories it is necessary to study triples of these histories and forms of triangles with their sides equal to distances between these histories. It turns out that some of these triangles will be proper, or isosceles with the small base. The portion of such triangles is just the ultrametricity degree. If this degree is equal to one, the set has a simple tree-like hierarchical structure. So the degree of ultrametricity is characterizing the degree of hierarchical organization of the history set.

By comparing ultrametrical characteristics of the history sets (Ezhov, Khrennnikov, \& Terentyeva, 2008), it was found, that for these two kinds of agents, the both coding schemes were complementary if the inequality in presenting food to different identity cells is not too high (Figure 3, Figure 4). This reflects a high symmetry between these memory codes. But if this inequality exceeds some threshold value, phase transition occurs and these two coding schemes preserve their symmetry only if the $\{A, R\}$ scheme is attributed to the right brain agents and the $\{\mathrm{S}, \mathrm{G}\}$ scheme to the left brain agents. It means that the symmetry observed at a lower inequality level has be- comes lower (Figure 4).

One can give an interesting interpretation of this phenomenon and explain why the $\{\mathrm{A}, \mathrm{R}\}$ coding becomes specific for the right brain agents which attract each other, and the $\{S, G\}$ coding-for the left brain agents which repulse each other (Figure 3). Recall that the agents solve a contradictory problem: they try to hold the both resources. By definition, the left brain agents are those which try to accept the environment proposal (to consume food as agents), i.e. to enhance the first (physical) resource. On the other hand, the right brain agents try to hold their current identity cell in order to save their second (mental) resource.

In this sense, they possess built-in automatic strategies which permit them to hold the first and the second resources, correspondingly. By doing so, they, however, risk to lose their uncontrolled resource (the second for the left brain agents and the first for the right brain ones). From this point of view, it will be extremely useful for the agents to memorize the cases of loosing or receiving the second (for the left brain agents) and the first (for the 


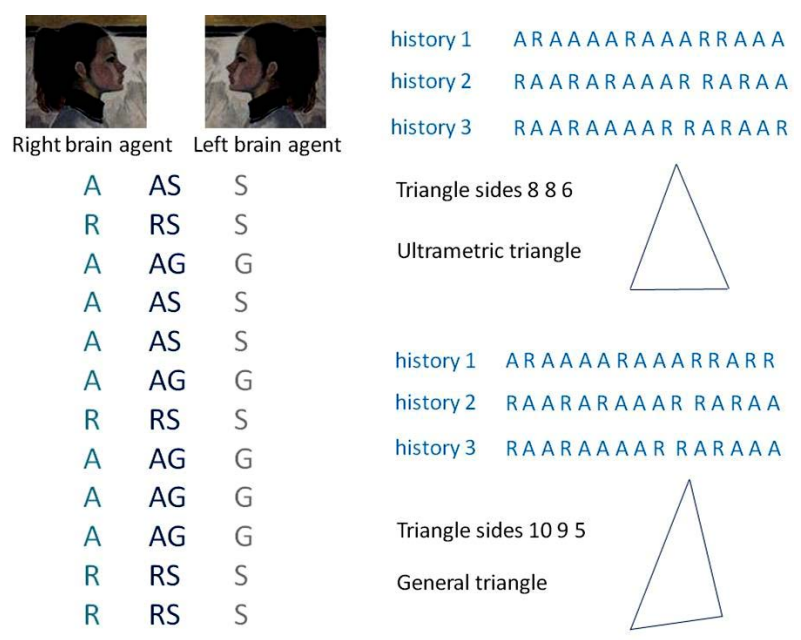

Figure 3. (Left) The full three binary code $\{A S, R S, A G\}$ is split into two incomplete binary codes $\{A, R\}$ and $\{S, G\}$ which become specific for the right and left brain agents in the situation of strong inequality. (Right) Ultrametric properties of the binary-coded history sets can be found by calculating the portion of the ultrametric triangles composed from all history triples. The sides of these triangles are equal to the Hamming distances between histories (number of distinct symbols). The ultrametric triangles are either equilateral or isosceles with the small base (6 in the case shown on the figure top).
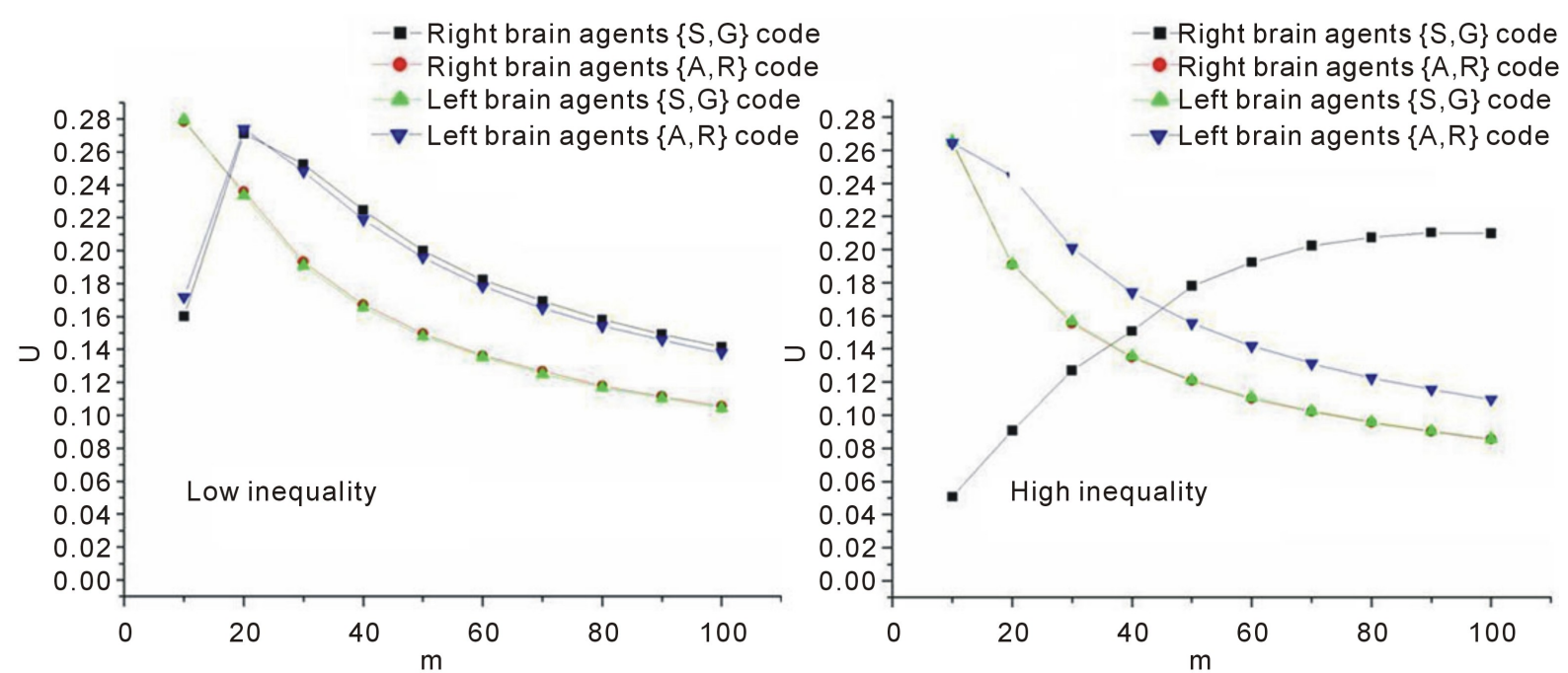

Figure 4. (Left) For low inequality the degree of ultrameticity (U) for the set of right brain agent histories of the length $m$ coded using the $\{A, R\}$ code coincides with those of the left brain agents histories coded using the $\{S, G\}$ code and vice versa. (Right) For a high inequality the degree of ultrameticity (U) for the set of right brain agent histories coded using the $\{\mathrm{A}$, $\mathrm{R}\}$ code coincides with those of left brain agents histories coded using the $\{\mathrm{S}, \mathrm{G}\}$ code but not vice versa! The symmetry is broken and specific codes appear.

right brain ones) resources or count them in order to control their quantity.

It is of interest enough, that for the right brain agents this corresponds to the act of memorizing the cases where they accepted or rejected the environment proposal (to consume or not to consume food). On the contrary, for the left brain agents it corresponds to the act of memorizing the cases where they stayed in the current cell or went to another cell. In fact, it just corresponds to the use of the $\{A, R\}$ code in the memory of the right brain agents and the $\{S, G\}$ code in the memory of the left brain agents.

Of course, the given agent-based models of artificial societies cannot prove or disprove anything. But they can show us some possibilities which can be consequences of the simplest properties of real societies. Indeed, the model described in (Ezhov \& Khrennikov, 2005: p. 016138-1) has been lately used by some physicians to explain the behavior of real patients suffering from alcoholism. It is evident that the duality of memory, as well 
as the process of counting was really observed in different periods of this disease (Nikonov, 2010: p. 11). Hence, we have some experimental evidence in favor of the view point that different memories are located in different hemispheres. Since one of the brain hemisphere is dominant in the given time, it is also true for one of the two parts of human memory. Note, that a binary structure is a very common phenomenon. In physics it is the presence of two basic types of particles—bosons and fermions, in mathematical psychology—-two basic ethic systems (Lefebvre, 2001).

\section{Cognitive Technology of Counting}

The emergence of the specific memory codes in a highly unequal society is considered to be the basis for simple cognitive technology of counting. This is also considered relevant to some observations conducted on patients suffered from alcoholism (Nikonov, 2011: p. 2). During the period of their disease-alcohol abstinent syndrome ("AAS") the patients implicitly count negative points corresponding with the moments of their behavior which hurt their relatives. After escaping the "AAS”, such patients tried to compensate the collected negative sum by doing good deeds and, hence, they had counted positive points. However, when it seems that the negative impression has been already compensated, it turns out that their relatives still remember that past unhappy time and unable to forgive. As a result, people addicted to alcohol are captured to "AAS" again. This provokes us to present a rather speculative and risky analogy of this phenomenon with the situation in the West-East relations during the latest centuries. In some sense, West had "suffered" from a certain kind of "AAS" and collected too many negative points until the collapse of the colonial system occured. After getting rid of this state in the 20th century, West tried to compensate those centuries of violence and dominance by giving a variety of preferences to emigrants from former colonies. Nevertheless, later, to a great surprise, it has turned out that no payment for the previous crimes was enough because the memory of them still existed. It may happen that certain contemporary events in Europe reveal its moving to a new traumatic period of "AAS".

\section{Memory Structures}

Now let us try to ask whether the results of agent-based model studies (Ezhov \& Khrennnikov, 2005) can be used for real brain modeling. Specific codes found in the model studies are attributed to two different agent types, i.e. the left and right brain dominants. We can guess that they can correspond to the codes of two hemispheres of the single model brain. Now it is now widely recognized that human memory is not unitary but composed of distinct systems localized in different brain structures and, at least, it can have dual character. The Dual-memory theories introduced different dichotomies such as a declarative/procedure knowledge, explicit/ implicit memories, etc. (now it is clear that the picture is more complicated and memory has a multiple character (Squire, 2004). The dual theory with a declarative/procedure (or non-declarative) dichotomy attributed them to the hippocampus of the medial temporal lobe and to the striatum of the basal ganglia, correspondingly (Squire, 1992). Moreover declarative and non-declarative memories are functionally independent and have a fundamentally different character (Krupa, 2009). However these memories can interact with each other. Different theories presume that this interaction in fact should have rather competitive than cooperative character (Krupa, 2009). In fact, memory mechanisms in the hippocampus and striatum competitively interact with each other at the response level after the process of learning has already occurred (Krupa 2009).

It is important that different memory systems differ not only in the locus of the competitive interaction, but also in their ability to be modulated by external factors such as distraction. This suggests that the locus of competition, be it at the level of acquisition or response, could influenced by external factors as well (Krupa, 2009). Moreover, “...current research is limited in that it does not explain how various social, environmental, and physiological parameters influence the interaction between the two competing memory systems (Poldrack \& Packard, 2003)”. The model (Ezhov \& Khrennikov, 2005) indeed is able, in principle, to offer a tool that allows this influence to be taken into account. In fact it employs two memory systems - the first uses the code $(S, G)$ and can be treated as the novelty detective ("Go" corresponds to transition to a new niche) and the second one registers accepting and rejection ("Reject") of the proposal. We can accept the hippocampus as the candidate brain structure for implementing the first function (or amygdala - research of Blackford et al. (Blackford, 2010). showed that only amygdala is distinctly responsive to novel unusual stimuli). On the other hand, the prefrontal cortex that is involved in the decision-making (Wood \& Grafman, 2003) can be treated as a structure for the recording acception or rejection. Indeed the experimental studies carried by D. Knoch et al. (Knoch, 2006) showed 
that just the magnetic field stimulation of the right dorsolateral prefrontal cortex suppresses the rejection of an unfair proposal in the ultimatum game. Since the $\{A, R\}$ code becomes specific for the right brain dominant agent, we can speculate that in certain conditions of high inequality the left hippocampus can be the site for the $\{\mathrm{S}, \mathrm{G}\}$ coding of novelty. The hypothesis about presentation of memory in the prefrontal cortex (PFC) can be supported by considerations also presented in (Wood \& Grafman, 2003). The PFC underlines many of higher cognitive functions such as language, reasoning, planning and social behavior. Wood and Grafman (Wood \& Grafman, 2003) argued that apart from these processes performed by PFC it also plays important representational role in brain. The authors (Wood \& Grafman, 2003) define the representations as "memories that are localized in neural networks that encode information and, when activated, enable access to this stored information". They wrote:

"Our structured event complex (SEC) framework proposes that the PFC stores unique forms of knowledge... An SEC is a goal-oriented set of events that is structured in sequence and represents thematic knowledge, morals, abstractions, concepts, social rules, event features, event boundaries and grammars”.

This indicates a possible relation between PFC and the history representations considered in the agent-base model. We propose that the dorsolateral PFC (DLPFC) can be considered as a location of memory coded by using the $\{\mathrm{A}, \mathrm{R}\}$ code. Though DLPFC has no direct connection to the hippocampus (treated as a location of memory coded using the $\{\mathrm{S}, \mathrm{G}\}$ code. It can interact with the last via ventromedial PFC. The model (Ezhov \& Khrennikov, 2005) suggests that in the situation of high inequality (unfairness) the $\{A, R\}$ code be specific for the right brain agents while the $\{\mathrm{S}, \mathrm{G}\}$ code-for the left brain ones. Then one can guess that in this situation the right DLPFC becomes a winner in the competition with the right hippocampus, while the left hippocampus dominates over the left DLPFC (Figure 5). Some analogy of this situation is observed, e.g., in a major depressive disorder characterized by a hypoactivity of the left DLPFC and also by a hyperactivity of the right DLPFC (Grimm et al., 2008).

\section{Conclusion}

It is possible to use either experimental or theoretical tools to study memory mechanisms. The number of experimental tools is constantly growing and along with EEG and PET includes such new tools as MEG, fMRI, transcranial magnetic simulation, etc. On the other hand, there also exist a number of theoretical approaches to describe memory mechanisms, such as e.g. connectionism, holography, and even quantum mechanics (Ricciardi \& Umezawa, 1967). Here we have argued that in principle the agent-based models are also suitable for theoretical treatment of the brain memory, especially in the case of influence of external sociological factors such as inequality. Note, that recently the agent-based approach has been successfully used (David-Barrett \& Dunbar,

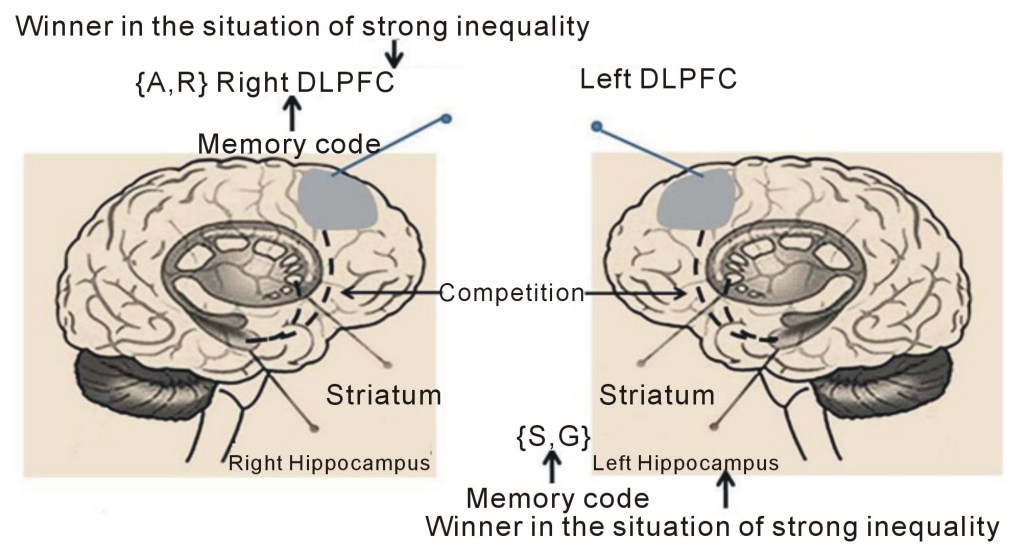

Figure 5. Two competitive systems in two brain hemispheres are shown. The known hippocampus-striatum (short black interrupted arc) and the hypothetical hippocampus-PFC (longer black interrupted arc). Under high inequality in this hypothetical competitive pair PFC dominates over the hippocampus in the right hemisphere and uses the specific $\{\mathrm{A}, \mathrm{R}\}$ code to represent memory records. On the other hand, the hippocampus dominates over PFC in the left hemisphere and uses the $\{\mathrm{S}, \mathrm{G}\}$ code to represent memory records. 
2013a) to describe the emergence of both social stratification and elite cliques. In the other paper (David-Barrett \& Dunbar, 2013b) the agent-based model has also been used to confirm the statement of the social brain theory that big brain is necessary to live in a big social group. We hope that our model gives another example of the possible using an idea of use agent-base modeling in the social brain theory to solve such a problem as the emergence of codes of memory in the brain structures of human being living in an unequal society.

\section{Acknowledgements}

We are very grateful to Dr. Michelle Y. Kibby for her kindly invitation to submit a paper to the Special Issue on "Mechanisms of Memory". We are also thankful to N.A. Milovidova for her assistance in preparing this paper.

\section{References}

Blackford, J. U., Buckholtz, J. W., Avery, S. N., \& Zald, D. H. (2010). A Unique Role for the Human Amygdala in Novelty Detection. NeuroImage, 50, 1188-1193. http://dx.doi.org/10.1016/j.neuroimage.2009.12.083

David-Barrett, D., \& Dunbar, R. I. M. (2013a). Social Elites Can Emerge Naturally When Interaction in Networks Is RESTRICTED. Behavioral Ecology, 25, 58-68. http://dx.doi.org/10.1093/beheco/art085

David-Barrett, D., \& Dunbar, R. I. M. (2013b). Processing Power Limits Social Group Size: Computational Evidence for the Cognitive Costs of Sociality. Proceedings of the Royal Society B, 22.

Easaw, J., McKay, A., \& Savoia, A. (2010). Inequality, Democracy and Institutions. World development, 38, 142-154.

Ezhov, A. A., \& Khrennikov, A. Yu. (2005). Agents with Left and Right Dominant Hemispheres and Quantum Statistics. Physical Review E, 71, 1-8.

Ezhov, A. A., \& Khrennikov, A. Yu. (2006). On Ultrametricity and a Symmetry between Bose-Einstein and Fermi-Dirac Systems. AIP Conference Proceedings, 826, 55-64. http://dx.doi.org/10.1063/1.2193110

Ezhov, A. A., Khrennikov, A. Yu., \& Terentyeva, S. S. (2008). Indication of a Possible Symmetry and Its Breaking in a Agent-Based Model Obeying Quantum Statistics. Physical Review E, 77, 1-12.

Grimm, S., Beck, J., Schuepbach, D., Hell, D., Boesiger, P., Bermpohl, F., Niehaus, L., Boeker, H., \& Northoff, G. (2008). Imbalance between Left and Right Dorsolateral Prefrontal Cortex in Major Depression Is Linked to Negative Emotional Judgment: An fMRI Study in Severe Major Depressive Disorder. Biological Psychiatry, 63, 369-376.

http://dx.doi.org/10.1016/j.biopsych.2007.05.033

Guckenheimer, J., \& Ottino, J. M. (2008). Foundations for Complex Systems Research in the Physical Sciences and Engineering. Report from NSF Workshop.

Knoch, D., Gianotti, L. R., Pasquale-Leone, A., Treyer, V., Regard, M., Hohmann, M., \& Brugger, P. (2006). Disruption of Right Prefrontal Cortex by Low-Frequency Repetitive Transcranial Magnetic Simulation Induces Risk-Taking Behavior. The Journal of Neuroscience, 26, 6469-6472. http://dx.doi.org/10.1523/JNEUROSCI.0804-06.2006

Krupa, A. K. (2009). The Competitive Nature of Declarative and Nondeclarative Memory Systems: Converging Evidence from Animal and Human Brain Studies. UCLA Undergraduate Science Journal, 22, 39-46.

Lefebvre, V. A. (2001). Algebra of Conscience. Dordrecht, London: Kluwer Academic Publisher. http://dx.doi.org/10.1007/978-94-017-0691-9

Lin, L., Osan, R., \& Tsien, J. (2006). Organizing Principles of Real-Time Memory Encoding: Neural Clique Assemblies and Universal Neural Codes. Trends in Neurosciences, 29, 48-57. http://dx.doi.org/10.1016/j.tins.2005.11.004

Mantegna, R. N., \& Stanley, H. E. (2000). An Introduction to Econophysics. Cambridge, England: Cambridge University Press.

Murtagh, F. (2006). Advances in Data Analysis. Berlin: Springer.

Nikonov, Yu. V. (2010). Brain Hemisphere Asymmetry and Quantum Statistics in Alcohol Dependence. Asymmetry, 4, $12-23$.

Nikonov, Yu. V. (2011). Interhemispheric Asymmetry and Simulation of Codependency. Asymmetry, 5, 11-19.

Poldrack, R. A., \& Packard, M. G. (2003). Competition among Multiple Memory Systems: Converging Evidence from Animal and Human Brain Studies. Neuropsychologia, 41, 245-251. http://dx.doi.org/10.1016/S0028-3932(02)00157-4

Ricciardi, L. M., \& Umezawa, H. (1967). Brain Physics and Many-Body Problems. Kibernetik, 4, 44-48. http://dx.doi.org/10.1007/BF00292170

Rotenberg, V. S., \& Arshavsky, V. V. (1997). Right and Left Brain Hemispheres Activation in the Representatives of Two Different Cultures. Homeostasis, 38, 49-57.

Scafetta, N., Picozzi, S., \& West, B. J. (2004). An Out-of-Equilibrium Model of the Distribution of Wealth. Quantitative 
Finance, 4, 353-364. http://dx.doi.org/10.1088/1469-7688/4/3/010

Squire, L. R. (1992). Memory and the Hippocampus: A Synthesis from Findings with Rats, Monkeys, and Humans. Psychological Review, 99, 195-231. http://dx.doi.org/10.1037/0033-295X.99.2.195

Squire, L. R. (2004). Memory Systems of the Brain: A Brief History and Current Perspective. Neurobiology of Learning and Memory, 82, 171-177. http://dx.doi.org/10.1016/j.nlm.2004.06.005

Thaler, R. H. (1988). The Ultimatum Game. Journal of Economic Perspectives, 2, 195-206. http://dx.doi.org/10.1257/jep.2.4.195

Tricomi, E., Rangel, A., Camerer, C. F., \& O’Doherty, J. P. (2010). Neural Evidence for Inequality-Averse Social Preferences. Nature, 463, 1089-1091. http://dx.doi.org/10.1038/nature08785

Wood, J. N., \& Grafman, J. (2003). Human Prefrontal Cortex: Processing and Representational Perspectives. Nature Reviews. Neuroscience, 4, 139-147. http://dx.doi.org/10.1038/nrn1033 